\title{
Isopropylbenzene oxidation reaction computer simulation
}

\author{
M.K. Vovdenko ${ }^{1}$, I.M. Gubaidulin ${ }^{1,2}$, K.F. Koledina ${ }^{1,2}$, S.N. Koledin ${ }^{1,2}$ \\ ${ }^{1}$ Institute of Petrochemistry and Catalysis Russian Science Academy, Prospect Oktyabrya 141, 450075, Ufa, Russia \\ ${ }^{2}$ Ufa State Technological Petroleum University, Kosmonavtov street 1, 450062, Ufa, Russia
}

\begin{abstract}
Isopropylbenzene (cumene) oxidation by air oxygen is intermediate stage of phenol and acetone production in cumene method. Cumene method of synthesis is currently the most wide-spread in the world to produce these two chemicals. Reaction is radical-chained and there also unwanted byproducts received along with desired ones.

This reaction has been subject of study since 30th of XX century. Different authors propose different ways of chain reactions, initiation mechanisms and influence degree of different factors and parameters during process.

In this paper different kinetic models of this reaction are compared and results of computations are shown.
\end{abstract}

Keywords: isopropylbenzene; oxidation; phenol; acetone; radical-chain mechanism; mathematic modeling.

\section{Introduction}

Phenol and acetone are very important substances in today petrochemistry industry. Acetone is used in paints, varnishes and solvents production. Also it is used as intermediate substance in petrochemistry industry. Phenol is used as intermediate substance in big number of petrochemistry units, such as bisphenol-A (which is lately used for polycarbonate and epoxies production), phenolic resins production, etc. Currently world industry produces more than 7 million tons of phenol per year [1] and this number growth annually. More than $97 \%$ of pnenol in the world is produced from cumene method and isopropylbenzene oxidation [1].

\section{Isopropylbenzene oxidation reaction computer simlation}

First cumene type production units were built at $40^{\text {th }}$ years of XX century in USSR (Russia) and Canada [2], but more detailed studies of process kinetics and mathematical model appeared in $60-70^{\text {th }}$ of XX century [3,4,5,6]. Because of low calculation machines capacities of that time researchers tried to simplify kinetics and mathematical models making different assumptions.

The general scheme of substances conversion is shown on Fig. 1 [7]:

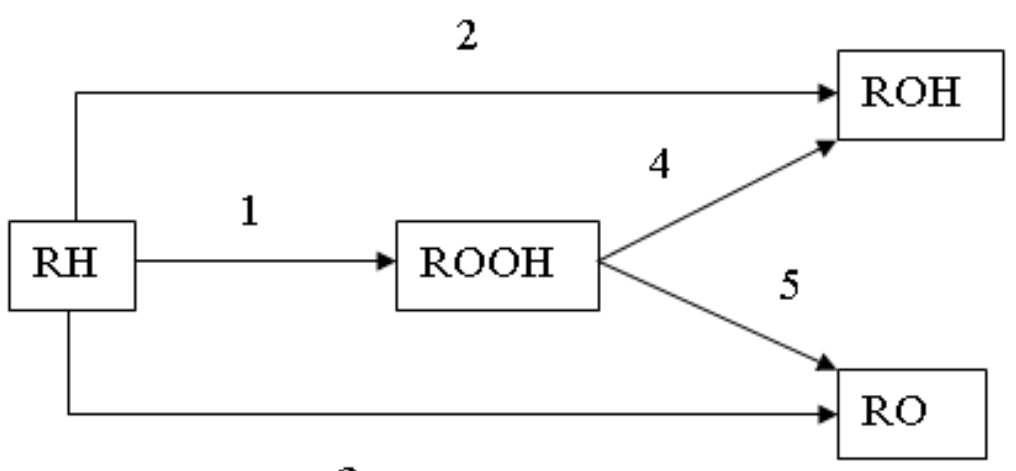

3

Fig. 1. General reactions scheme.

$\mathrm{R} \bullet$ is cumyl radical $\left(\mathrm{C}_{6} \mathrm{H}_{5}\left(\mathrm{CH}_{3}\right)_{2} \mathrm{C} \bullet\right.$, $\mathrm{RH}$ is cumene $\left(\mathrm{C}_{6} \mathrm{H}_{5}\left(\mathrm{CH}_{3}\right)_{2} \mathrm{CH}\right)$, $\mathrm{ROOH}$ is cumene hydroperoxide $\left(\mathrm{C}_{6} \mathrm{H}_{5}\left(\mathrm{CH}_{3}\right)_{2} \mathrm{COOH}\right)$, $\mathrm{ROH}$ - dymethylphenylcarbinol (DMPC is $\left.\mathrm{C}_{6} \mathrm{H}_{5}\left(\mathrm{CH}_{3}\right)_{2} \mathrm{COH}\right)$, $\mathrm{RO}$ is acetophenone $\left(\mathrm{C}_{6} \mathrm{H}_{5} \mathrm{CH}_{3} \mathrm{CO}\right)$.

But in reality this reactions follow radical-chain mechanism. There are number of elementary steps on which decomposition of particles and radicals recombination happens. Let's look at Hattori radical-chain reaction mechanism and on reaction scheme from [6] as examples [3].

Where In is initiator, which decomposes on radicals with an easy, so overall reaction process is enhanced. In some experiments author used benzene hydroperoxide as initiator [3] but we studied experiment without any additional initiator. Cumene hydroperoxide itself is used as initiator in most production units and laboratory researches. So reactions of stage 1 (1a and $1 \mathrm{~b}$ ) are replaced by $3 \mathrm{a}, 3 \mathrm{~b}, 3 \mathrm{c}$ reactions for this case.

B1 and B2 are some substances which can be detected exactly (different organic acids, aldehydes, alcohols). 
Computer Modeling / M.K. Vovdenko, I.M. Gubaidulin, K.F. Koledina, S.N. Koledin Table 1. Hattori cumene oxidation kinetics model [3].

\begin{tabular}{|c|c|c|}
\hline Reaction number & Reaction & Constant \\
\hline $1 \mathrm{a}$ & $\mathrm{In}_{2} \rightarrow 2 \mathrm{In} \bullet$ & - \\
\hline $1 \mathrm{~b}$ & $\mathrm{In} \bullet+\mathrm{RH} \rightarrow \mathrm{R} \bullet+\mathrm{InH}$ & - \\
\hline $2 a$ & $\mathrm{RH} \rightarrow \mathrm{R} \bullet+\mathrm{H} \bullet$ & $\mathrm{k} 2 \mathrm{a}$ \\
\hline $2 b$ & $\mathrm{RH}+\mathrm{O}_{2} \rightarrow \mathrm{R} \bullet+\mathrm{HO}_{2} \bullet$ & $\mathrm{k} 2 \mathrm{~b}$ \\
\hline $3 a$ & $\mathrm{ROOH} \rightarrow \mathrm{RO} \bullet+\cdot \mathrm{OH}$ & $\mathrm{k} 3 \mathrm{a}$ \\
\hline $3 b$ & $\mathrm{RO} \bullet+\mathrm{RH} \rightarrow \mathrm{R} \bullet+\mathrm{ROH}$ & $\mathrm{k} 3 \mathrm{~b}$ \\
\hline $3 \mathrm{c}$ & $\cdot \mathrm{OH}+\mathrm{RH} \rightarrow \mathrm{R} \bullet+\mathrm{H}_{2} \mathrm{O}$ & $\mathrm{k} 3 \mathrm{c}$ \\
\hline $4 a$ & $\mathrm{R} \bullet+\mathrm{O}_{2} \rightarrow \mathrm{RO}_{2} \bullet$ & $\mathrm{k} 4 \mathrm{a}$ \\
\hline $4 \mathrm{~b}$ & $\mathrm{RO}_{2} \bullet+\mathrm{RH} \rightarrow \mathrm{R} \bullet+\mathrm{ROOH}$ & $\mathrm{k} 4 \mathrm{~b}$ \\
\hline $5 \mathrm{a}$ & $2 \mathrm{R} \cdot \rightarrow \mathrm{R}-\mathrm{R}$ & $\mathrm{k} 5 \mathrm{a}$ \\
\hline $5 b$ & $\mathrm{R} \bullet+\mathrm{RO}_{2} \bullet \rightarrow \mathrm{ROOR}$ & $\mathrm{k} 5 \mathrm{~b}$ \\
\hline $5 \mathrm{c}$ & $2 \mathrm{RO}_{2} \bullet \rightarrow \mathrm{ROOR}+\mathrm{O}_{2}$ & $\mathrm{k} 5 \mathrm{c}$ \\
\hline $6 a$ & $\mathrm{RO} \bullet \rightarrow\left(\mathrm{C}_{6} \mathrm{H}_{5}\right) \mathrm{CH}_{3} \mathrm{CO}+\mathrm{CH}_{3} \bullet$ & $\mathrm{k} 6 \mathrm{a}$ \\
\hline $6 \mathrm{~b}$ & $\mathrm{CH}_{3} \bullet+\mathrm{O}_{2} \rightarrow \mathrm{CH}_{3} \mathrm{O}_{2} \bullet$ & $\mathrm{k} 6 \mathrm{~b}$ \\
\hline $6 c$ & $\mathrm{CH}_{3} \mathrm{O}_{2} \bullet+\mathrm{RH} \rightarrow \mathrm{HCHO}+\mathrm{H}_{2} \mathrm{O}+\mathrm{R} \bullet$ & $\mathrm{k} 6 \mathrm{c}$ \\
\hline
\end{tabular}

Table 2. Cumene oxidation kinetics scheme [6].

\begin{tabular}{|c|c|c|}
\hline Reaction number & Reaction & Constant \\
\hline 0 & $\mathrm{RH}+\mathrm{O}_{2} \rightarrow \mathrm{R} \bullet+\mathrm{HO}_{2} \bullet$ & $\mathrm{k} 0$ \\
\hline 1 & $\mathrm{R} \bullet+\mathrm{O}_{2} \rightarrow \mathrm{RO}_{2} \bullet$ & $\mathrm{k} 1$ \\
\hline 2 & $\mathrm{RO}_{2} \bullet+\mathrm{RH} \rightarrow \mathrm{ROOH}+\mathrm{R}^{\bullet}$ & $\mathrm{k} 2$ \\
\hline 3 & $\mathrm{ROOH}+\mathrm{RH} \rightarrow \mathrm{RO} \bullet+\mathrm{R} \bullet+\mathrm{H} 2 \mathrm{O}$ & $\mathrm{k} 3$ \\
\hline 4 & $2 \mathrm{ROOH} \leftrightarrow[\mathrm{ROOH}]_{2}$ & $\mathrm{k} 4$ \\
\hline 5 & {$[\mathrm{ROOH}]_{2} \rightarrow \mathrm{RO}_{2} \bullet+\mathrm{RO} \bullet+\mathrm{H}_{2} \mathrm{O}$} & $\mathrm{k} 5$ \\
\hline 6 & $\mathrm{RO} \bullet+\mathrm{RH} \rightarrow \mathrm{ROH}+\mathrm{R} \bullet$ & $\mathrm{k} 6$ \\
\hline 7 & $\cdot \mathrm{OH}+\mathrm{RH} \rightarrow \mathrm{H}_{2} \mathrm{O}+\mathrm{H}_{2} \mathrm{O}$ & $\mathrm{k} 7$ \\
\hline 8 & $\mathrm{RO} \bullet \rightarrow \mathrm{R}^{\prime} \mathrm{O}+\mathrm{CH}_{3} \bullet$ & $\mathrm{k} 8$ \\
\hline 9 & $\mathrm{CH}_{3} \bullet+\mathrm{RH} \rightarrow \mathrm{CH}_{4}+\mathrm{R} \bullet$ & $\mathrm{k} 9$ \\
\hline 10 & $\mathrm{CH}_{3} \bullet+\mathrm{O}_{2} \rightarrow \mathrm{CH}_{3} \mathrm{OO} \bullet$ & $\mathrm{k} 10$ \\
\hline 12 & $\mathrm{CH}_{3} \mathrm{OO} \bullet+\mathrm{RH} \rightarrow \mathrm{CH}_{3} \mathrm{OOH}+\mathrm{R} \bullet$ & $\mathrm{k} 11$ \\
\hline 13 & $\mathrm{H}_{2} \mathrm{CO}+\mathrm{R} \bullet \rightarrow \mathrm{HC} \bullet \mathrm{O}+\mathrm{R} \bullet$ & $\mathrm{k} 12$ \\
\hline 14 & $\mathrm{HC} \cdot \mathrm{O}+\mathrm{O}_{2} \rightarrow \mathrm{HCOOO} \bullet$ & $\mathrm{k} 13$ \\
\hline 15 & $\mathrm{HCOOO} \bullet+\mathrm{RH} \rightarrow \mathrm{HCOOOH}+\mathrm{R} \bullet$ & k14 \\
\hline 16 & $\mathrm{HCOOOH} \rightarrow \mathrm{HCOO} \bullet+\cdot \mathrm{OH}$ & $\mathrm{k} 15$ \\
\hline 17 & $\mathrm{HCOO} \bullet+\mathrm{RH} \rightarrow \mathrm{HCOOH}+\mathrm{R} \bullet$ & k16 \\
\hline 18 & $\mathrm{HCOOO} \bullet \rightarrow \mathrm{CO} 2+\bullet \mathrm{OH}$ & $\mathrm{k} 17$ \\
\hline 19 & $2 \mathrm{RO}_{2} \bullet \rightarrow 2 \mathrm{R}^{\prime} \mathrm{O}+2 \bullet \mathrm{CH}_{3}+\mathrm{O}_{2}$ & $\mathrm{k} 18$ \\
\hline 20 & $2 \mathrm{RO}_{2} \bullet \rightarrow \mathrm{B} 1$ & k19 \\
\hline 21 & $\mathrm{RO}_{2} \bullet+\mathrm{R} \bullet \rightarrow \mathrm{B} 2$ & $\mathrm{k} 20$ \\
\hline
\end{tabular}

For Hattori reaction scheme simulation we made equations system based on Table 1 [8] where rate reactions are unknown:

$$
\begin{aligned}
& W_{2 a}=k 2 a \cdot[R H] \\
& W_{2 b}=k 2 b \cdot[R H] \cdot\left[O_{2}\right] \\
& W_{3 a}=k 3 a \cdot[R O O H] \\
& W_{3 b}=k 3 b \cdot[R O \cdot][R H] \\
& W_{3 c}=k 3 c \cdot[\cdot O H][R H] \\
& W_{4 a}=k 4 a \cdot[R \cdot] \cdot\left[O_{2}\right]
\end{aligned}
$$




$$
\begin{aligned}
& W_{4 b}=k 4 b \cdot\left[R O_{2} \cdot\right][R H] \\
& W_{5 a}=k 5 a \cdot[R \cdot]^{2} \\
& W_{5 b}=k 5 b \cdot[R \cdot]\left[R O_{2} \cdot\right] \\
& W_{5 c}=k 5 c \cdot\left[R O_{2} \cdot\right]^{2} \\
& W_{6 a}=k 6 a \cdot[R O \cdot] \\
& W_{6 b}=k 6 b \cdot\left[\mathrm{CH}_{3} \cdot\right] \cdot\left[O_{2}\right] \\
& W_{6 c}=k 6 c \cdot\left[\mathrm{CH}_{3} \mathrm{O}_{2} \cdot\right][R H] \\
& W_{6 d}=k 6 d \cdot[R O \cdot][R H]
\end{aligned}
$$

Then we created system of differential equitation's, in which reagents concentration changing throw time is shown $[3,8]$ :

$$
\begin{aligned}
& \frac{d[R H]}{d t}=-k 2 a \cdot[R H]-k 2 b \cdot[R H]-k 3 b \cdot[R O \cdot][R H]-k 3 c \cdot[\cdot O H][R H]-k 4 b \cdot\left[R O_{2}\right][R H]- \\
& -k 6 c \cdot\left[\mathrm{CH}_{3} \mathrm{O}_{2} \cdot\right][\mathrm{RH}]-k 6 d \cdot[\mathrm{RO} \cdot][\mathrm{RH}] \\
& \frac{d[\mathrm{ROOH}]}{d t}=-k 3 a \cdot[\mathrm{ROOH}]+k 4 b \cdot\left[\mathrm{RO}_{2}\right][\mathrm{RH}] \\
& \frac{d[R \cdot]}{d t}=k 2 a \cdot[R H]+k 2 b \cdot[R H]+k 3 b \cdot[R O \cdot][R H]-k 4 a \cdot[R \cdot]+k 4 b \cdot\left[R O_{2}\right][R H]-k 5 a \cdot[R \cdot]^{2}- \\
& -k 5 b[R \cdot]\left[\mathrm{RO}_{2}\right]+k 6 c \cdot\left[\mathrm{CH}_{3} \mathrm{O}_{2} \cdot\right][\mathrm{RH}]+k 6 d \cdot[\mathrm{RO} \cdot][\mathrm{RH}] \\
& \frac{d\left[R O_{2} \cdot\right]}{d t}=-k 4 b \cdot\left[R O_{2} \cdot\right][R H]+k 4 a \cdot[R \cdot]-k 5 b \cdot[R \cdot]\left[R O_{2} \cdot\right]-k 5 b \cdot[R \cdot]\left[R O_{2} \cdot\right] \\
& \frac{d[R O \cdot]}{d t}=-k 3 b \cdot[R O \cdot][R H]+k 3 a \cdot[R O O H]-k 6 a \cdot[R O \cdot]-k 6 d \cdot[R O \cdot][R H] \\
& \frac{d[\cdot O H]}{d t}=k 3 a \cdot[R O O H]-k 3 c \cdot[\cdot O H][R H] \\
& \frac{d[R-R]}{d t}=k 5 b \cdot[R \cdot]\left[R O_{2} \cdot\right]+k 5 c \cdot\left[R O_{2} \cdot\right]^{2} \\
& \frac{d[R O O R]}{d t}=-k 2 a \cdot[R H]-k 2 b \cdot[R H]-k 3 b \cdot[R O \cdot][R H]-k 3 c \cdot[\cdot O H][R H]-k 4 b \cdot\left[R O_{2}\right] \\
& \frac{d[\mathrm{H} 2 \mathrm{O}]}{d t}=k 3 c \cdot[\cdot \mathrm{OH}][\mathrm{RH}]+k 6 \mathrm{c} \cdot\left[\mathrm{CH}_{3} \mathrm{O}_{2} \cdot\right][\mathrm{RH}]+k 6 d \cdot[\mathrm{RO} \cdot][\mathrm{RH}] \\
& \frac{d[R O H]}{d t}=k 3 b \cdot[R O \cdot][R H] \\
& \frac{d[H \cdot]}{d t}=k 2 a \cdot[R H] \\
& \frac{d[\mathrm{HO} 2 \cdot]}{d t}=k 2 b \cdot[R H] \\
& \frac{d[\mathrm{cH} 3]}{d t}=k 6 a \cdot[R O \cdot]-k 6 b \cdot\left[\mathrm{CH}_{3} \cdot\right] \\
& \frac{d\left[\mathrm{CH}_{3} \mathrm{O}_{2} \cdot\right]}{d t}=k 6 b \cdot\left[\mathrm{CH}_{3} \cdot\right]-k 6 c \cdot\left[\mathrm{CH}_{3} \mathrm{O}_{2} \cdot\right][\mathrm{RH}] \\
& \frac{d[\mathrm{HCHO}]}{d t}=k 6 c \cdot\left[\mathrm{CH}_{3} \mathrm{O}_{2} \cdot\right][\mathrm{RH}] \\
& \frac{d[(\mathrm{C} 6 \mathrm{H} 5) \mathrm{CCH} 3 \mathrm{CH} 2]}{d t}=k 6 d \cdot[R O \cdot][R H]
\end{aligned}
$$

In order to solve this equitation system we used MATLAB program. In fact we need to solve reverse kinetic problem [9] which means that we need to find chemical rates reactions constants for given experimental data. We used data from [3,6,7]. On Fig. 2 graphs of concentration are shown, both for experimental and calculated data for experiment in [3].

\section{Conclusion}

In this paper different cumene oxidation mechanism were shown and calculation experiment was conducted. 


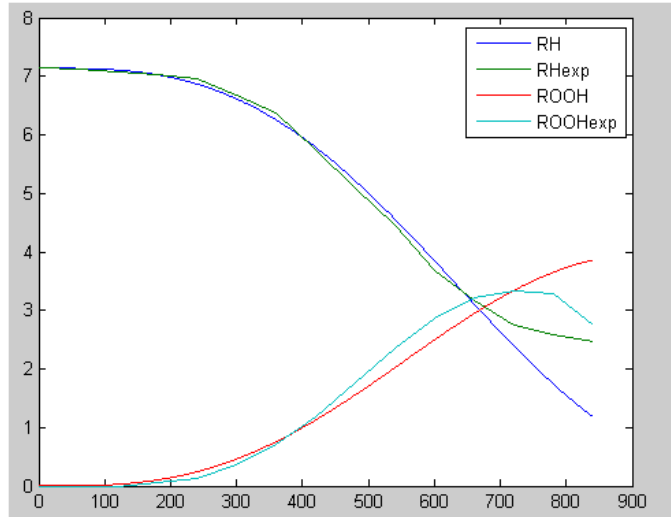

Fig. 2. MATLAB calculations result and experimental data [3].

\section{References}

[1] Zakoshansky VM. Alternative technologies of phenol and acetone production. Russian Journal of Chemistry 2008; LII(4): 53-71.

[2] ] Zakoshansky VM. Phenol and acetone: Technologies, kinetics and main reactions mechanisms analyziz. SPb.: Khimizdat, 2009.

[3] Hattori K, Tanaka Y, Suzuki H. Kinetics of liquid phase oxidation of cumene in bubble column. Journal of chemical Engineering of Japan 1970; 3(1): 72-78.

[4] Andrigo P, Caimi A, Cavalieri d'Oro P, Fiat A, Roberti L, Tampieri M, Tartari V. Phenol-acetone process: cumene oxidation kinetics and industrial plant simulation. Chemical Engineering Science 1992; 47(9-11): 2511-2516.

[5] Dale GH. Rate Constants of Osidation of Cumene. Journal of the American Chemical Society 1967; 89(21): 5433-5438.

[6] Makalec BI, Kirichenko GS, Stryigin EI. Liquid-phase model of cumene oxidation to hydroperoxide. Petrochemistry 1978; 18(2): $250-255$.

[7] Dahnavi EM, Ryazanov IG, Hardampidi HE. Temperature influence on catalytic cumene oxidation. Journal of Kazan Technological University 2009; 6: $263-266$.

[8] Gubaidullin IM. Informational and analytic system of inverse kinetic tasks: tutorial . Ufa: Published in BSU, 2003; 89 p.

[9] Koledian KF, Gubaidullin IM. Program complex for inverse kinetic tasks solution and its realisation as virtual test stend. Science and education: science edition MSTU N.E. Baumana 2013; 7: 385-398. 